\title{
Evidence-Based Selection of Candidates for the Levonorgestrel Intrauterine Device (IUD)
}

\author{
Lisa S. Callegari, MD, MPh, Blair G. Darney, PhD, MPh, Emily M. Godfrey, MD, MPh, \\ Olivia Sementi, MD, Rebecca Dunsmoor-Su, MD, MSCE, \\ and Sarab W. Prager, MD, MAS
}

Background: Recent evidence-based guidelines expanded the definition of appropriate candidates for the levonorgestrel-releasing intrauterine system (LNG-IUS). We investigated correlates of evidencebased selection of candidates for the LNG-IUS by physicians who offer insertion.

Methods: We conducted a mixed-mode (online and mail) survey of practicing family physicians and obstetrician-gynecologists in Seattle.

Results: A total of 269 physicians responded to the survey (44\% response rate). of the 217 respondents who inserted intrauterine devices, half or fewer routinely recommended the LNG-IUS to women who are nulliparous, younger than 20 years old, or have a history of sexually transmitted infections (STIs). In multivariable analyses, training/resident status was positively associated with recommending the LNG-IUS to women <20 years old (adjusted odds ratio [aOR], 3.6; 95\% confidence interval [CI], 1.6-8.0) and women with history of STI (aOR, 3.7; 95\% CI, 1.6-8.4). Perceived risk of infection or infertility was negatively associated with recommending the LNG-IUS to nulliparous women (aOR, 0.2 ; 95\% CI, 0.1-0.5) and women with a history of STI (aOR, 0.3; 95\% CI, 0.1-0.8).

Conclusions: Many family physicians and obstetrician-gynecologists who insert the LNG-IUS are overly restrictive in selecting candidates, although those who train residents are more likely to follow evidence-based guidelines. Interventions that address negative bias and perceptions of risks, in addition to improving knowledge, are needed to promote wider use of the LNG-IUS. (J Am Board Fam Med 2014;27:26-33.)

Keywords: Contraception, Evidence-based Medicine, Graduate Education, Intrauterine Devices

Nearly half of pregnancies in the United States are unintended, and $48 \%$ of these occur in women who report using contraception during the month in which the pregnancy occurred. ${ }^{1,2}$ Intrauterine de-

This article was externally peer reviewed.

Submitted 5 May 2013; revised 20 August 2013; accepted 26 August 2013.

From the Departments of Obstetrics and Gynecology (LSC, OS, RD-S, SWP) and Family Medicine (EMG) and the Departments of Epidemiology (LSC) and Department of Health Services (SWP), School of Public Health, University of Washington, Seattle; the Departments of Medical Informatics and Clinical Epidemiology and Obstetrics and Gynecology (BGD), Oregon Health \& Science University, Portland.

Funding: Dr. Callegari received support from the US Department of Health and Human Services, Health Resources and Services Administration's Maternal and Child Health Bureau (Title V, Social Security Act; grant no. T76MC00011). Dr. Darney received support from a predoctoral training grant (TL1 RR025016) and an Agency for Healthcare Research and Quality postdoctoral training grant (T32 HS017582). vices (IUDs) are a safe, highly effective, and longacting contraceptive option with high acceptability among women. ${ }^{3,4}$ A recent large, prospective study demonstrated that IUDs are 20 times more effective in preventing pregnancy than other commonly used hormonal methods such as the birth control pill. ${ }^{5}$ Despite these advantages, however, IUDs remain underused in the United States compared with other industrialized countries. ${ }^{6,7}$

IUDs that are currently available in the United States include the copper T380A IUD, introduced

Prior presentation: Presented at the 60th Annual Clinical Meeting of the American College of Obstetrics and Gynecology, San Diego, California, May 6-9, 2012.

Conflict of interest: Dr. Godfrey has received trainer honoraria from Merck Pharmaceuticals and advisory board honoraria from Teva Women's Health.

Corresponding author: Lisa S. Callegari, MD, MPh, Seattle HSR\&D Center, University of Washington, 1100 Olive Way, \#1400, Seattle, WA 98101 (E-mail: lcallega@uw.edu). 
in 1988; levonorgestrel intrauterine system (LNGIUS), which releases $20 \mu \mathrm{g}$ levonorgestrel/day and was introduced in 2000; and a version of the LNGIUS that releases $14 \mu \mathrm{g} /$ day and was approved by the US Food and Drug Administration (FDA) in January 2013. In the family planning community, growing attention has focused on expanding the use of IUDs containing levonorgestrel in particular, given their high effectiveness and acceptability as well as their noncontraceptive benefits. ${ }^{8}$ A large body of evidence investigating the $20-\mu \mathrm{g} /$ day LNG-IUS demonstrates a range of clinical benefits including a reduction in menstrual flow and anemia, ${ }^{9-11}$ reduction in dysmenorrhea, ${ }^{12}$ and reduction in the risk of endometrial hyperplasia and cancer. ${ }^{13}$

Health care providers have a critical role to play in expanding use of IUDs by both providing accurate information and following evidence-based guidelines when offering the method to eligible candidates. ${ }^{6}$ IUDs have traditionally been viewed as best suited to older multiparous women, and product labeling for the $20-\mu \mathrm{g} /$ day LNG-IUS still recommends limiting use to women who have had a least one child. ${ }^{14}$ Current evidence-based guidelines, including the Centers for Disease Control and Prevention's 2010 US Medical Eligibility Criteria for Contraceptive $\mathrm{Use}^{15}$ and the American College of Obstetrics and Gynecology practice recommendations, ${ }^{7,16}$ however, state that all IUDs can be safely used by nulliparous women, adolescents, and women with a history of sexually transmitted infections (STIs), pelvic inflammatory disease (PID), or ectopic pregnancy. The American College of Obstetrics and Gynecology recommends that IUDs be considered first-line contraceptive options for most women, including nulliparous and adolescent women. ${ }^{7,16}$

Prior studies have described high rates of inaccurate perceptions of IUD risks and overly restrictive views of candidates eligible for IUD use by family physicians and obstetrician-gynecologists. ${ }^{17-22} \mathrm{Sev}-$ eral recent surveys of family physicians and obstetrician-gynecologists investigated predictors of whether providers perform IUD insertions and found that important factors include accurate knowledge of IUD risks, IUD training during residency, and "expansive" or evidence-based views on eligible candidates. $^{20,22}$ To date, however, published studies have not examined correlates of following current evidence-based guidelines in selecting candidates eli- gible for IUDs by those family physicians and obstetrician-gynecologists who perform insertions.

Therefore, we surveyed family physicians and obstetrician-gynecologists who insert IUDs to identify factors associated with routinely recommending the LNG-IUS to women not traditionally viewed as eligible or not eligible according to package labeling but who are considered appropriate candidates on the basis of current evidence-based guidelines. We investigated associations between demographic, practice, knowledge, and attitude factors and evidence-based selection of candidates for the LNG-IUS. We focused on those physicians who already insert IUDs, given that interventions to expand use of the LNG-IUS in this population are likely to have the most impact in expanding access in the short term, and on the LNG-IUS in particular because of its multiple advantages, including its noncontraceptive benefits. Our survey was completed before introduction of the $14-\mu \mathrm{g} /$ day LNG-IUS; therefore, our results pertain only to the $20-\mu \mathrm{g} /$ day LNG-IUS.

\section{Methods \\ Data Collection}

We conducted a mixed-mode (online and mail) survey of all identified family physicians and obstetrician-gynecologists, including specialists and generalists, practicing in Seattle, Washington, assessing their knowledge of, attitudes toward, and practices related to the LNG-IUS. We identified potential subjects using the Washington State Medical Association Directory, hospital directories, local phone books, and Internet searches. We then sent an E-mail with an invitation to complete an online survey to all potential subjects with an identified E-mail address. For email survey nonresponders, up to $2 \mathrm{E}$-mail reminders were sent. We mailed a paper survey to those potential subjects without an identified E-mail address. Recipients of the paper survey could either complete and send back the paper version or follow instructions to access the online version. Recipients of the paper survey were asked to send in a separate prestamped postcard indicating that they had completed the paper or online survey. Paper surveys were returned anonymously with no subject identifiers. One reminder was sent to those recipients of the paper survey who did not return a postcard indicating that they had completed the survey. No 
incentive was provided. All respondents were practicing in Seattle and spoke English. The study was granted institutional review board exemption by the University of Washington. The study was unfunded.

\section{Survey}

The 21-question survey was based on a questionnaire used in a previous study of physician knowledge of, attitudes toward, and practices related to the Copper T IUD ${ }^{21}$ and was modified to focus on the LNG-IUS. Physician characteristics assessed included gender, specialty (obstetrics and gynecology or family practice), practice setting (academic/ private/public health/other), whether the physician trained residents or fellows, and whether the physician completed residency $\geq 10$ years ago. Physicians were asked, "Which choice below most accurately reflects your current approach to IUD use?" and chose 1 of 3 response options: (1) "recommend to no one," (2) "recommend to selected patients but refer to others for insertion," and (3) "recommend and insert
IUDs." We considered a physician to insert IUDs if he or she selected option 3. Physicians also were asked to identify the number of LNG-IUSs they place per year $(0,1-10,11-20,>20)$.

Figure 1 summarizes the survey questions assessing physician knowledge, attitudes, and practice. We assessed general knowledge of the LNGIUS using 2 multiple-choice questions concerning the LNG-IUS's failure rate and FDA-approved length of use. We included additional knowledge questions regarding 5 hypothetical complications of LNG-IUS: whether the LNG-IUS is associated with risk of infertility after removal (correct answer is no), risk of PID $<20$ days after insertion (correct answer is yes), risk of PID $>20$ days after insertion (correct answer is no), and risk of acquiring an STI or experiencing ectopic pregnancy (correct answer is no for both). We assessed attitudes toward the LNG-IUS by asking about factors that could negatively influence a provider's choice to recommend the LNG-IUS, including a perceived increased risk of PID, STIs, or infertility with LNG-IUS use,

Figure 1. Knowledge, attitude, and practice survey questions. Mirena is the trade name for releasing levonorgestrel intrauterine system releasing $20 \mu \mathrm{g} /$ day. IUD, intrauterine device; PID, pelvic inflammatory disease; STI, sexually transmitted infection. FDA, US Food and Drug Administration.

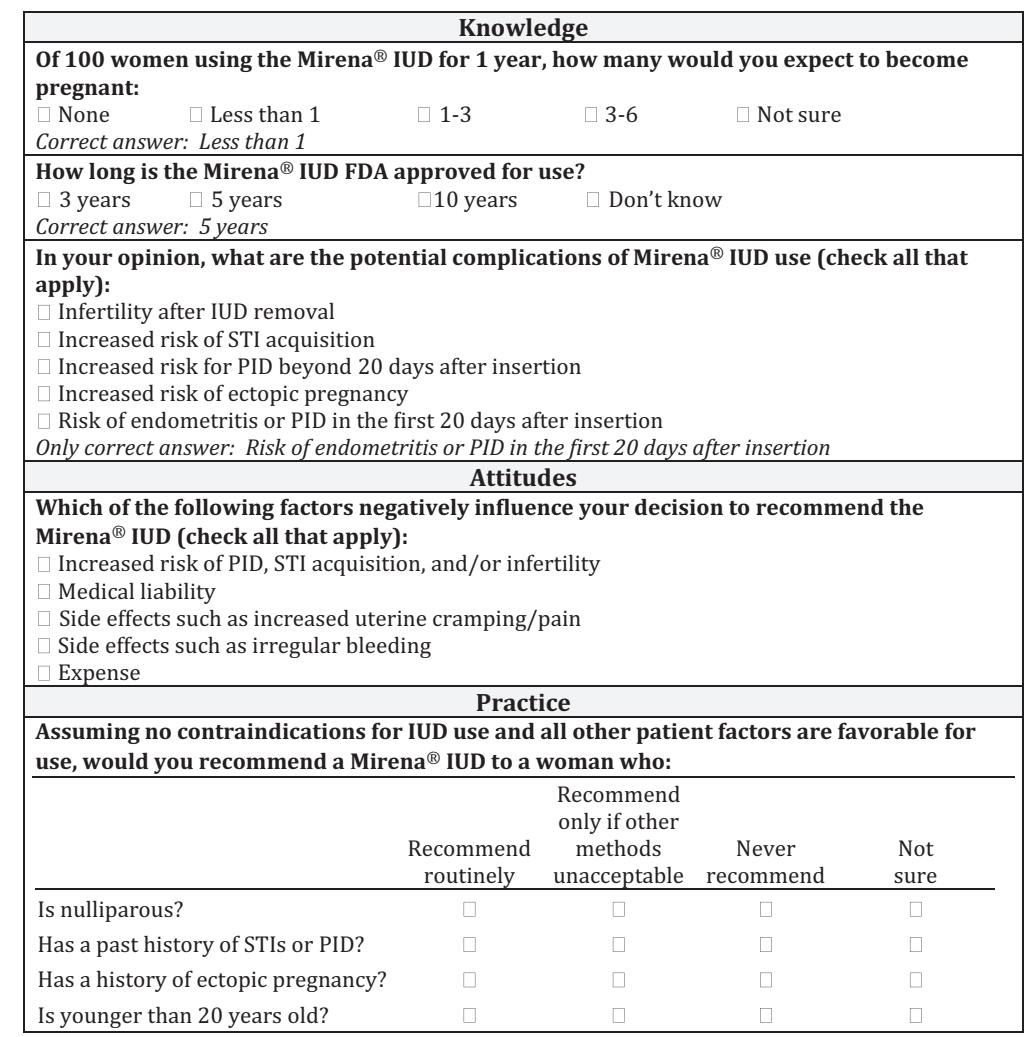


concern about the patient experiencing cramping or pain upon insertion, or concern that the patient could experience irregular bleeding as a side effect. Providers also were asked whether concerns about medical liability or cost to the patient were negative influences in their decisions about whether to recommend the LNG-IUS.

We assessed physician practices relating to selection of eligible candidates by asking whether providers would routinely recommend the LNGIUS assuming "no contraindications for IUD use and all other patient factors are favorable for use" among 4 types of patients: (1) nulliparous women, (2) women $<20$ years old, (3) women with a history of STIs or PID, and (4) women with a history of ectopic pregnancy (Figure 1). We refer to these practices as using "evidence-based" selection of LNG-IUS candidates based on recommendations from the Centers for Disease Control and Prevention's 2010 Medical Eligibility for Contraceptive Use. $^{15}$

\section{Data Analysis}

Because we focused on selection of LNG-IUS candidates by physicians who insert IUDs, we excluded respondents from our sample who reported that they did not insert IUDs. We performed a descriptive analysis of physicians who insert IUDs. Our primary study outcomes were binary measures of whether a physician reported that he or she routinely recommends the LNG-IUS to the 4 patient types listed above, assuming all other conditions are favorable for IUD use. We developed 4 multivariable logistic regression models to assess factors associated with routinely recommending the LNGIUS to each of the 4 patient types.

We selected model covariates a priori by their potential importance to LNG-IUS practices based on a literature review. ${ }^{23}$ Potential factors included demographic and practice characteristics and knowledge of and perceptions about the LNG-IUS. ${ }^{17-21,24,25}$ Demographic and practice factors included specialty, practice setting, whether the provider completed training $\geq 10$ years ago, gender, involvement in training residents or fellows, and whether the provider inserted $\geq 10$ LNG-IUSs per year. In the multivariable models we considered providers to have accurate general knowledge if they provided correct answers to both general knowledge questions. For accurate knowledge related to complications of the LNG-IUS, we included a score of complications knowledge ranging from 1 to 5 , calculated by the number of correct responses to the 5 questions about complications of the LNG-IUS described earlier. We excluded medical liability as a negative influence because only $1.8 \%$ of respondents answered that this had a negative influence on their practice. We retained in the models all other variables defined a priori as covariates regardless of statistical significance testing. ${ }^{23}$ All analyses were conducted using Stata software version 10.0 (StataCorp, LP, College Station, TX).

\section{Results}

Of the 641 surveys sent by E-mail or mail, 32 surveys were not delivered to the intended recipient because of an incorrect addresses. A total of 269 physicians responded to the survey, including 125 online surveys and 144 paper surveys, with a $44 \%$ overall response rate. Our study sample comprised the 217 respondents who reported that they insert IUDs (Table 1). Of the sample, $61 \%$ was family physicians, $72 \%$ was female, and $56 \%$ practiced in a private setting. Two thirds of respondents $(65 \%)$ reported that they trained residents or fellows; all physicians practicing in an academic setting (43 of 43 ), and $57 \%$ of physicians (97 of 169) in nonacademic settings were involved in training residents or fellows (data not shown). In addition, $62 \%$ of the sample had finished residency training $\geq 10$ years before the survey and $44 \%$ inserted $\geq 10$ LNGIUSs per year.

Only $75 \%$ of respondents correctly identified both the LNG-IUS failure rate and FDA-approved length of use (Table 1). Most respondents correctly answered that infertility, increased risk of PID $\geq 20$ days after insertion, and increased risk of STI acquisition are not complications of LNG-IUS use (95\%, $91 \%$, and $90 \%$, respectively). However, despite these high levels of knowledge, 22\% reported that concern about STIs, PID, or risk of infertility with the LNG-IUS negatively influenced their decision to recommend the LNG-IUS.

In the multivariable logistic regression models (Table 2), physician specialty (family medicine vs obstetrics-gynecology) was not associated with evidence-based selection of LNG-IUS candidates. Teaching residents or fellows was positively associated with routine recommendation of the LNGIUS to adolescents (adjusted odds ratio [aOR] 3.56; 
Table 1. Sample Characteristics of Physicians Who Insert the Levonorgestrel Intrauterine System (LNG-IUS) $(\mathbf{n}=217)$

\begin{tabular}{|c|c|}
\hline \multicolumn{2}{|l|}{ Demographic and practice characteristics } \\
\hline Female gender & $157(72.4)$ \\
\hline \multicolumn{2}{|l|}{ Specialty } \\
\hline Family physician & $132(60.8)$ \\
\hline Obstetrician-gynecologist & $85(39.2)$ \\
\hline \multicolumn{2}{|l|}{ Practice setting } \\
\hline Academic & $43(19.8)$ \\
\hline Private practice & $122(56.2)$ \\
\hline Public health & $47(21.7)$ \\
\hline Trains residents or fellows & $141(65.0)$ \\
\hline$\geq 10$ years since residency & $134(61.8)$ \\
\hline$\geq 10$ LNG-IUSs inserted/year & $96(44.2)$ \\
\hline \multicolumn{2}{|l|}{ Knowledge } \\
\hline \multicolumn{2}{|l|}{ General, answered correctly } \\
\hline LNG-IUS failure rate & $177(81.6)$ \\
\hline LNG-IUS FDA-approved duration of use & $198(91.2)$ \\
\hline Perfect score on 2 general knowledge items & $163(75.1)$ \\
\hline \multicolumn{2}{|l|}{ Complications, answered correctly } \\
\hline Infertility after removal & $206(94.9)$ \\
\hline $\begin{array}{l}\text { Increased risk for PID } \geq 20 \text { days after } \\
\text { insertion }\end{array}$ & $198(91.2)$ \\
\hline $\begin{array}{l}\text { Increased risk for PID }<20 \text { days after } \\
\text { insertion }\end{array}$ & $125(57.6)$ \\
\hline Increased risk of STI & $195(89.9)$ \\
\hline Increased risk of ectopic pregnancy & $123(56.7)$ \\
\hline $\begin{array}{l}\text { Complications score, mean number } \\
\text { answered correctly out of } 5(\mathrm{SD})^{*}\end{array}$ & $3.9(0.93)$ \\
\hline \multicolumn{2}{|l|}{ Attitudes } \\
\hline \multicolumn{2}{|l|}{$\begin{array}{l}\text { Factors that negatively influence decision to } \\
\text { recommend LNG-IUS, answered yes }\end{array}$} \\
\hline Increased risk of PID/STIs/infertility & $48(22.1)$ \\
\hline Cramping/pain side effect & $25(11.5)$ \\
\hline Irregular bleeding side effect & $69(31.8)$ \\
\hline Medical liability & $4(1.8)$ \\
\hline Cost & $47(21.7)$ \\
\hline \multicolumn{2}{|l|}{ Practice } \\
\hline \multicolumn{2}{|l|}{$\begin{array}{l}\text { Routinely recommend LNG-IUS for the } \\
\text { following patients, answered yes }\end{array}$} \\
\hline Nulliparous women & $121(55.8)$ \\
\hline Women with a history of STIs/PID & $82(37.8)$ \\
\hline Women with a history of ectopic pregnancy & $81(37.3)$ \\
\hline Women $<20$ years old & $80(36.9)$ \\
\hline
\end{tabular}

Data are n (\%).

${ }^{*}$ Complications score is calculated from number of correct answers on the 5 questions listed.

PID, pelvic inflammatory disease; SD, standard deviation; STI, sexually transmitted infection; FDA, US Food and Drug Administration.

95\% confidence interval [CI], 1.58-8.04), women with a history of STIs or PID (aOR, 3.71; 95\% CI, 1.64-8.43), and women with a history of ectopic pregnancy (aOR, 3.05; 95\% CI, 1.32-7.01). We observed a trend toward association of teaching with routine recommendation of the LNG-IUS to nulliparous women that did not achieve statistical significance (aOR, 1.95; 95\% CI, 0.88-4.30). The associations were not limited to academic centers because practice setting was not independently associated with evidence-based selection of candidates; training residents was positively associated with evidence-based use of the LNG-IUS regardless of practice setting.

Perceived increased risk of infertility, STIs, or PID was negatively associated with routine recommendation of the LNG-IUS to nulliparous women (aOR 0.19; 95\% CI, 0.08-0.45), women with a history of STIs or PID (aOR 0.30; 95\% CI, 0.110.77 ), and women with a history of ectopic pregnancy (aOR 0.38; 95\% CI, 0.15-0.98). An association in the same direction was present with routine recommendation to adolescents but was not statistically significant (aOR 0.85; 95\% CI, 0.36-2.00). A correct score on both basic knowledge items was positively associated with routine recommendation to nulliparous women (aOR 2.39; 95\% CI, 1.09-5.25), whereas having completed residency $\geq 10$ years ago and perceived concern about irregular bleeding were negatively associated $(\mathrm{aOR}, 0.44 ; 95 \% \mathrm{CI}$, 0.21-0.93; and aOR, 0.40; 95\% CI, 0.18-0.88, respectively). Inserting $>10$ LNG-IUSs per year was associated with routine recommendation for women with a history of STIs or PID (aOR, 2.28; 95\% CI, 1.04-5.01).

\section{Discussion}

Providers have an important role to play in making long-acting reversible contraception such as the LNG-IUS accessible to patients, yet many physicians who insert the LNG-IUS do not follow evidence-based guidelines when selecting eligible candidates. We found only one half or fewer of the physicians in our sample routinely recommend the LNG-IUS to women who are nulliparous, $<20$ years old, or have a history STIs, PID, or ectopic pregnancy. Involvement in teaching residents or fellows was positively associated with evidencebased selection of candidates, whereas factors such as practice setting and physician specialty were not associated. Physicians with negative perceptions about infection-related risks of using LNG-IUS were less likely to practice evidence-based selection of candidates. 
Table 2. Factors Associated With Physicians Routinely Recommending the $20-\mu \mathrm{g} / \mathrm{day}$ Levonorgestrel Intrauterine System (LNG-IUS) to 4 Patient Types: Multivariable Logistic Regression Models

\begin{tabular}{|c|c|c|c|c|}
\hline & \multicolumn{4}{|c|}{ Patient Types* } \\
\hline & $\begin{array}{l}\text { Nulliparous } \\
\text { Women }\end{array}$ & $\begin{array}{l}\text { Adolescents } \\
(\text { age }<20)\end{array}$ & $\begin{array}{l}\text { Women With a } \\
\text { History of } \\
\text { STIs or PID }\end{array}$ & $\begin{array}{l}\text { Women With a } \\
\text { History of } \\
\text { Ectopic Pregnancy }\end{array}$ \\
\hline \multicolumn{5}{|l|}{$\begin{array}{l}\text { Physician demographics and practice } \\
\text { characteristics }\end{array}$} \\
\hline Family physician $^{\dagger}$ & $1.59(0.68-3.57)$ & $1.45(0.66-2.78)$ & $1.72(0.77-3.85)$ & $0.53(0.24-1.16)$ \\
\hline Academic practice setting $^{\ddagger}$ & $1.32(0.51-3.46)$ & $0.99(0.43-2.30)$ & $0.97(0.40-2.33)$ & $0.75(0.31-1.85)$ \\
\hline Greater than 10 years since residency & $0.44(0.21-0.93)^{\S}$ & $0.73(0.37-1.45)$ & $0.76(0.37-1.53)$ & $0.93(0.45-1.91)$ \\
\hline Female sex & $2.14(0.97-4.74)$ & $0.92(0.43-1.96)$ & $0.90(0.40-2.01)$ & $1.24(0.56-2.79)$ \\
\hline Trains residents/fellows & $1.95(0.88-4.30)$ & $3.56(1.58-8.04)^{\S}$ & $3.71(1.64-8.43)^{\S}$ & $3.05(1.32-7.01)^{\S}$ \\
\hline More than 10 LNG-IUS inserted per year & $1.50(0.67-1.53)$ & $1.50(0.70-3.20)$ & $2.28(1.04-5.01)^{\S}$ & $1.11(0.51-2.42)$ \\
\hline \multicolumn{5}{|l|}{ Knowledge about LNG-IUS } \\
\hline Perfect score on 2 item general knowledge & $2.39(1.09-5.25)^{\S}$ & $1.62(0.73-3.60)$ & $1.57(0.69-3.56)$ & $1.15(0.51-2.61)$ \\
\hline Complications score ${ }^{\|}$(continuous out of 5) & $1.02(0.68-1.53)$ & $1.11(0.76-1.63)$ & $0.96(0.64-1.43)$ & $1.84(1.18-2.88)^{\S}$ \\
\hline \multicolumn{5}{|l|}{$\begin{array}{l}\text { Perceived factors that negatively influence } \\
\text { recommending LNG-IUS, answered yes }\end{array}$} \\
\hline Cost & $0.76(0.33-1.74)$ & $0.44(0.19-1.02)$ & $1.39(0.60-3.21)$ & $0.92(0.39-2.16)$ \\
\hline Irregular bleeding & $0.40(0.18-0.88)^{\S}$ & $1.42(0.66-3.04)$ & $0.56(0.25-1.24)$ & $1.30(0.59-2.87)$ \\
\hline Cramping/pain & $1.04(0.33-3.29)$ & $0.56(0.18-1.69)$ & $0.33(0.09-1.20)$ & $0.30(0.08-1.11)$ \\
\hline Risk of infertility/STIs/PID & $0.19(0.08-0.45)^{\S}$ & $0.85(0.36-2.00)$ & $0.30(0.11-0.77)^{\S}$ & $0.38(0.15-0.98)^{\S}$ \\
\hline
\end{tabular}

Data are adjusted odds ratios ( $95 \%$ confidence intervals).

${ }^{*}$ Logistic regression models adjust for all variables in the table ( $\mathrm{n}=186$ for all models).

${ }^{\dagger}$ Reference is obstetrician-gynecologist.

${ }^{\ddagger}$ Reference is nonacademic practice settings.

${ }^{\S} P<.05$.

"The complications score is calculated from number of correct answers on 5 questions regarding infertility, PID, STI, and ectopic pregnancy risks of LNG-IUS use.

PID, pelvic inflammatory disease; STI, sexually transmitted infection.

Our descriptive findings join those of multiple other studies in highlighting overly restrictive selection of candidates for use of the LNGIUS. ${ }^{17,18,20-22,25}$ In their 2012 national survey of family physicians and obstetrician-gynecologists, Harper et $\mathrm{al}^{22}$ similarly found that only a third of respondents would recommend IUD to teenagers, half to nulliparous women, and a third to women with history of STIs, PID, or ectopic pregnancy. In a national survey of office-based and Title $\mathrm{X}$ clinic providers, Tyler et $\mathrm{al}^{25}$ found that a comparably low percentage of providers (40\%) routinely provided the LNG-IUS to nulliparous women.

The results from our 4 multivariable regression models provide insights into factors associated with evidence-based selection of LNG-IUS candidates. First, involvement in teaching residents or fellows was associated with 3 -fold increased odds of routinely recommending the LNG-IUS to adolescents, women with a history of STIs or PID, and women with a history of ectopic pregnancy. Teach- ing was associated with 2-fold increased odds of routinely recommending the LNG-IUS to nulliparous women, but the association did not reach statistical significance. The observed associations were independent of practice setting (academic vs nonacademic). To our knowledge, no prior studies examining IUD use have considered the role of a provider's involvement in teaching. ${ }^{17-22}$ Our findings could indicate an "up-training" effect whereby trainees bring new evidence and innovations into established practice, or it could reflect that physicians who choose to be involved in teaching are more likely to follow current guidelines. Further research is needed to better understand the role of teaching in the adoption of evidence-based guidelines.

Second, in our multivariable models negative perceptions of the risk of STIs, PID, or infertility with LNG-IUS use was associated with overly restrictive selection of LNG-IUS candidates, independent of physicians' level of knowledge. In addition, our descriptive results showed that while most 
respondents in our sample answered correctly that infertility, STIs, and PID beyond 20 days after insertion were not complications of LNG-IUS use, a significant number still reported that concerns about risk of infection negatively influenced their practice. These findings suggest that changing practice may require addressing physician biases that persist despite accurate knowledge. Previous work in the practice change literature suggests that translating evidence into clinical practice requires more than disseminating information or improving knowledge. ${ }^{19,26,27}$ In the contraception literature, Kohn et $\mathrm{al}^{28}$ identified a similar discrepancy between knowledge and behavior in a survey of school-based health center providers about their knowledge and attitudes toward the provision of IUDs to teens. In that survey, providers reported accurate knowledge about the safety of IUDs in teens but often remained unwilling to recommend IUDs to teens. Using qualitative interviews with primary care physicians, Rubin et $\mathrm{al}^{29}$ developed a conceptual framework of factors influencing IUD practices related to teens. Highlighting the independent roles of both accurate knowledge and perceptions of the risks and benefits of IUDs, they concluded that interventions should address both knowledge gaps and negative attitudes and be tailored to individual practice sites. Our findings also suggest that further study of the complex interactions between knowledge, perceptions, and bias related to IUD practices is needed to inform the development of effective practice change interventions.

Several other associations observed in the multivariable models deserve mention. First, the estimated association between having completed residency $\geq 10$ years ago and routinely recommending the LNG-IUS was negative for all 4 models but was significant only for nulliparous women. The direction of these associations is consistent with studies indicating that physicians farther from their training are less likely to insert IUDs ${ }^{18}$ and more likely to have misperceptions about IUDs ${ }^{25}$; however, the lack of significance in 3 models suggests either a lack of a true association or insufficient sample size to detect significance. In a similar way, the direction of the associations between accurate general knowledge and routinely recommending the LNGIUS was positive in the 4 models but the association was significant only for nulliparous women; this may indicate that accurate knowledge alone does not consistently predict evidence-based practices ${ }^{28,29}$ or may be due to sample size. Further investigation in a larger sample is warranted to clarify these observed associations.

Our results should be interpreted with several limitations in mind. Response bias may be a concern, as with any survey study. Our response rate of $44 \%$, however, is not substantially different from other published literature on physician surveys. ${ }^{30,31}$ Given that responders may have been more interested in or knowledgeable about contraception than nonresponders, our results may overestimate general knowledge and evidence-based use of the LNG-IUS. In addition, the characteristics of providers who insert the LNG-IUS in Seattle may be different from providers in other cities, rural areas, or other regions of the country, which also limits the generalizability of our findings. For example, $61 \%$ of our sample was family physicians, which may be a higher percentage compared with other urban areas. We chose to focus our study on those providers who already insert the LNG-IUS, and therefore our results cannot be generalized to all providers who provide contraceptive care. Additional research is needed to address barriers to physicians inserting IUDs. A 2011 national survey of family physicians found that those who inserted more IUDs during residency were more likely to offer insertions in their practice. ${ }^{20}$ Interventions are needed to both expand the base of providers who offer insertion and expand the spectrum of patients considered eligible by providers who insert the LNG-IUS.

\section{Conclusion}

This study highlights both deficiencies in knowledge and the overly restrictive selection of LNGIUS candidates by both family physicians and obstetrician-gynecologists who insert IUDs, particularly among physicians who do not train residents or fellows. Even when providers had accurate knowledge regarding risks of infection with the LNG-IUS, many still reported perceived concern about risks of STIs, PID, and infertility with LNG-IUS use. Practice change interventions that address negative bias and perceptions as well as improve knowledge are needed to promote evidence-based practice and wider use of the LNG-IUS. 


\section{References}

1. Finer LB, Zolna MR. Unintended pregnancy in the United States: incidence and disparities, 2006. Contraception 2011;84:478-85.

2. Finer LB, Henshaw SK. Disparities in rates of unintended pregnancy in the United States, 1994 and 2001. Perspect Sex Reprod Health 2006;38:90-6.

3. Trussell J. Contraceptive failure in the United States. Contraception 2011;83:397-404.

4. Peipert JF, Zhao Q, Allsworth JE, et al. Continuation and satisfaction of reversible contraception. Obstet Gynecol 2011;117:1105-13.

5. Winner B, Peipert JF, Zhao Q, et al. Effectiveness of long-acting reversible contraception. N Engl J Med 2012;366:1998-2007.

6. Allen RH, Goldberg AB, Grimes DA. Expanding access to intrauterine contraception. Am J Obstet Gynecol 2009;201:456.e1-5.

7. American College of Obstetricians and Gynecologists Committee on Gynecologic Practice; Long-Acting Reversible Contraception Working Group. ACOG Committee Opinion no. 450: Increasing use of contraceptive implants and intrauterine devices to reduce unintended pregnancy. Obstet Gynecol 2009;114:1434-8.

8. Turok DK. What the world needs now is more access to the levonorgestrel IUD. Contraception 2013;87:391-2.

9. Lowe RF, Prata N. Hemoglobin and serum ferritin levels in women using copper-releasing or levonorgestrel-releasing intrauterine devices: a systematic review. Contraception 2013;87:486-96.

10. Kadir RA, Chi C. Levonorgestrel intrauterine system: bleeding disorders and anticoagulant therapy. Contraception 2007;75(6 Suppl):S123-9.

11. Pakarinen P, Luukkainen T. Treatment of menorrhagia with an LNG-IUS. Contraception 2007;75(6 Suppl):S118-22.

12. Gemzell-Danielsson K, Schellschmidt I, Apter D. A randomized, phase II study describing the efficacy, bleeding profile, and safety of two low-dose levonorgestrel-releasing intrauterine contraceptive systems and Mirena. Fertil Steril 2012;97:616-22.e1-3.

13. Hubacher D, Grimes DA. Noncontraceptive health benefits of intrauterine devices: a systematic review. Obstet Gynecol Surv 2002;57:120-8.

14. Mirena package insert. Wayne, NJ: Bayer HealthCare Pharmaceutials Inc; February 2013. Available from: http://labeling.bayerhealthcare.com/html/products/pi/ Mirena_PI.pdf. Accessed July 15, 2013.

15. Centers for Disease Control and Prevention (CDC). US medical eligibility criteria for contraceptive use, 2010. MMWR Recomm Rep 2010;59(RR-4):1-86.

16. Committee on Adolescent Health Care Long-Acting Reversible Contraception Working Group; American College of Obstetricians and Gynecologists. Committee opinion no. 539: adolescents and long- acting reversible contraception: implants and intrauterine devices. Obstet Gynecol 2012;120:983-8.

17. Stanwood NL, Garrett JM, Konrad TR. Obstetrician-gynecologists and the intrauterine device: a survey of attitudes and practice. Obstet Gynecol 2002; 99:275-80.

18. Madden T, Allsworth JE, Hladky KJ, Secura GM, Peipert JF. Intrauterine contraception in Saint Louis: a survey of obstetrician and gynecologists' knowledge and attitudes. Contraception 2010;81:112-6.

19. Harper CC, Blum M, de Bocanegra HT, et al. Challenges in translating evidence to practice: the provision of intrauterine contraception. Obstet Gynecol 2008;111:1359-69.

20. Rubin SE, Fletcher J, Stein T, Segall-Gutierrez P, Gold M. Determinants of intrauterine contraception provision among US family physicians: a national survey of knowledge, attitudes and practice. Contraception 2011;83:472-8.

21. Espey E, Ogburn T, Espey D, Etsitty V. IUDrelated knowledge, attitudes and practices among Navajo Area Indian Health Service providers. Perspect Sex Reprod Health 2003;35:169-73.

22. Harper CC, Henderson JT, Raine TR, et al. Evidence-based IUD practice: family physicians and obstetrician-gynecologists. Fam Med 2012;44:637-45.

23. Hosmer DW, Lemeshow S. Applied logistic regression. 2nd ed. New York: Wiley; 2000.

24. Kooiker CH, Scutchfield FD. Barriers to prescribing the Copper T 380A intrauterine device by physicians. West J Med 1990;153:279-82.

25. Tyler CP, Whiteman MK, Zapata LB, Curtis KM, Hillis SD, Marchbanks PA. Health care provider attitudes and practices related to intrauterine devices for nulliparous women. Obstet Gynecol 2012;119:762-71.

26. Grol R, Grimshaw J. From best evidence to best practice: effective implementation of change in patients' care. Lancet 2003;362:1225-30.

27. Cabana MD, Rand CS, Powe NR, et al. Why don't physicians follow clinical practice guidelines? A framework for improvement. JAMA 1999;282:1458-65.

28. Kohn JE, Hacker JG, Rousselle MA, Gold M. Knowledge and likelihood to recommend intrauterine devices for adolescents among school-based health center providers. J Adolesc Health 2012;51: 319-24.

29. Rubin SE, Davis K, McKee MD. New York City physicians' views of providing long-acting reversible contraception to adolescents. Ann Fam Med 2013; 11:130-6.

30. Cull WL, O'Connor KG, Sharp S, Tang SF. Response rates and response bias for 50 surveys of pediatricians. Health Serv Res 2005;40:213-26.

31. Dillman DA, Smyth JD, Christian LM. Internet, mail, and mixed-mode surveys: the tailored design method. 3rd ed. Hoboken, NJ: Wiley \& Sons; 2009. 